\title{
Concordance of the point-of-care circulating cathodic antigen test for the diagnosis of intestinal schistosomiasis in a low endemicity area
}

Sergei Rodrigo Magalhães de Sousa ${ }^{1,2^{*}}$ (D), Isabelle Helena Lima Dias ${ }^{1,2}$, Álvaro Luan Santana Fonseca ${ }^{1,2}$, Bianca Rodrigues Contente ${ }^{1,2}$, Joyce Favacho Cardoso Nogueira ${ }^{1,2}$, Tatyellen Natasha da Costa Oliveira ${ }^{1}$, Stefan Michael Geiger ${ }^{3}$ and Martin Johannes Enk ${ }^{1,2}$

\begin{abstract}
Background: The Kato-Katz technique is recommended worldwide for the diagnosis of intestinal schistosomiasis, detecting parasite eggs in feces of infected people. However, new tests have been developed in order to facilitate diagnosis, e.g. by detection of specific antigens secreted by schistosomes, such as the circulating cathodic antigen (CCA). The aim of this study was to evaluate the performance of the point-of-care circulating cathodic antigen test (POC-CCA) compared to the Kato-Katz technique in a low prevalence area in the Amazon Region, located in the municipality of Primavera, State of Pará, Brazil.

Methods: Positivity rates of the POC-CCA test and the Kato-Katz technique were calculated. The sensitivity, specificity, accuracy and kappa coefficient were determined by comparing both methods. The reference standard was established using 16 Kato-Katz slides, 12 of the first fecal sample, two of the second and two of the third one. The study also included the concordance between POC-CCA results and different numbers and combinations of Kato-Katz slides.

Results: The prevalence of schistosomiasis according to the reference standard or POC-CCA test reached a rate of $9.4 \%$ or $23.9 \%$, respectively, among a total of 372 participants. The positivity rates by the Kato-Katz technique increased from 2.4 to $9.4 \%$, according to the increase in the number of slides examined and fecal samples collected. A sensitivity of $55.6 \%$, specificity $76.9 \%$, accuracy $76 \%$ and $k$ coefficient of 0.06 was observed by comparing one slide of the first sample and POC-CCA. Comparing 6 slides from three different samples, two slides of each, with POC-CCA resulted in a sensitivity of $58.3 \%$, specificity $78.4 \%$, accuracy $77 \%$ and $k$ coefficient of 0.16 . Finally, the comparison of 16 slides from three different samples with POC-CCA revealed a sensitivity of $65.7 \%$, specificity $80.4 \%$, accuracy $79 \%$, and $k$ coefficient of 0.27 .

(Continued on next page)
\end{abstract}

\footnotetext{
* Correspondence: rodrigo.bio.uepa@gmail.com

${ }^{1}$ Programa de Pós-Graduação Strictu Sensu em Biologia Parasitária na Amazônia da Universidade do Estado do Pará, Tv. Perebebuí, 2623 - Marco, Belém, PA 66087, Brazil

${ }^{2}$ Laboratório de Parasitoses Intestinais, Esquistossomose e Malacologia (LPIEM), Secção de Parasitologia, Instituto Evandro Chagas/SVS/MS,

Ananindeua, Pará, Brazil

Full list of author information is available at the end of the article
}

(c) The Author(s). 2019 Open Access This article is distributed under the terms of the Creative Commons Attribution 4.0 International License (http://creativecommons.org/licenses/by/4.0/), which permits unrestricted use, distribution, and reproduction in any medium, provided you give appropriate credit to the original author(s) and the source, provide a link to the Creative Commons license, and indicate if changes were made. The Creative Commons Public Domain Dedication waiver (http://creativecommons.org/publicdomain/zero/1.0/) applies to the data made available in this article, unless otherwise stated. 


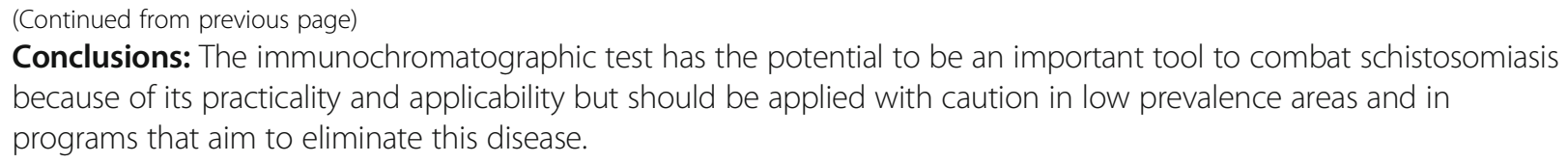

\section{Multilingual abstracts}

Please see Additional file 1 for translations of the abstract into the five official working languages of the United Nations.

\section{Background}

Schistosomiasis is a neglected disease of great matter in public health. The World Health Organization (WHO) organized WHA65.21 resolution adopted by endemic countries in World Health Assembly 2012, aimed to intensify the combat of this parasitic disease as a public health problem in endemic countries. The main objective is reducing positivity rate to less than $5.0 \%$ [1].

It is estimated that more than 206 million people required preventive treatment in 2016 to schistosomiasis around the world. Schistosomiasis transmission has been reported from 78 countries [2]. More than 1.5 million of these individuals are in risk areas in Brazil [3]. From 2010 to 2016, a total of 6233975 exams were performed, achieving endemic areas of Brazil, revealing a total of 273019 positives detected, with a positivity rate of $4.4 \%$ [4].

Currently, Kato-Katz technique is the recommended method for Schistosoma mansoni diagnosis [5], a quantitative and qualitative direct test besides low cost and high specificity, often underestimates positivity rates among infected individuals with low parasite burden in low infection rate areas [6-12].

Rapid methods, also called point of care (POC) tests, have been developing to facilitate schistosomiasis diagnostic. These immunochromatographic tests detect the presence of worm antigen in serum or urine, specifically circulating anodic antigen (CAA) and circulating cathodic antigen (CCA), respectively [13]. The advantage of using circulating antigens is the easy manipulation of urine samples in research field and the simple reading of results, not being necessary advanced training [14].

In 2015 WHO recommended the use both methods, POC-CCA and Kato-Katz in order to evaluate and monitor the control programs efficiency in endemic countries if the main goal is to eliminate schistosomiasis as a public health problem. Other methods are still being evaluated, as Helmintex ${ }^{\circ}$ test that has a great probability to become the "gold standard" to the diagnosis of schistosomiasis [15].
In Tanzania and Kenya, the POC-CCA test was used to identify $S$. mansoni antigen in urines from children, showing higher prevalence rates when compared to the Kato-Katz technique [16]. In Uganda this fast test has also shown better results when compared to two KatoKatz slides [17]. According to data from Europe, the application of POC-CCA among immigrants of endemic areas is quite encouraged, due to its practicality and high sensitivity [18]. Nowadays, the POC-CCA test is already being used to map S. mansoni infections in Africa [19].

Kittur et al. in a systematic review described a direct association between POC-CCA and Kato-Katz test results in areas with prevalence higher than $50 \%$ [20]. In areas with prevalence lower than $10.0 \%$, there is not enough evidence to evaluate the performance of the POC-CCA test [21]. In this point of view, this study aims to compare both diagnostic approaches in a low prevalence area, assessing the performance of the rapid test and its possible application in large scale.

\section{Methods}

\section{Population and study area}

This research was performed in the communities of Pedrinha and Canaã, municipalities of Primavera, located in the mesoregion of Northeastern Pará State, distant about $195 \mathrm{~km}$ from the capital Belém. The area contains 258.6 $\mathrm{km}^{2}$ and a population density of approximately 40 inhabi$\operatorname{tants} / \mathrm{km}^{2}$. The communities are part of the legal Amazon region, with tropical climate and temperatures ranging from $20^{\circ} \mathrm{C}$ to $40^{\circ} \mathrm{C}$. It presents climatic conditions where the annual average rainfall varies between $2500 \mathrm{~mm}$ and $3000 \mathrm{~mm}$ [22].

A total of 422 local residents were invited to participate of this research. Since the epidemiological study involved the whole population, there was no need for a representative sample size calculation. Previous surveys from Brazilian Schistosomiasis Control Program reported that the region is a low prevalence area, presenting positivity rates between 2.0 and $3.0 \%$.

Individuals who did not deliver three fecal and urine samples were excluded from the study. In addition, children less than 2 years old and pregnancy women were also excluded, which led to a reduction of the total population to 372 individuals. 


\section{Collection of biological samples}

Three fecal samples and three first morning urine samples were collected from the 372 remaining participants on three consecutive days (Fig. 1). For examination, the three fecal samples were processed while only one urine sample was used. The remaining urine samples were frozen and stored in a freezer at the Biobank of the Parasitology Section of the Instituto Evandro Chagas - Belém, Pará, Brazil.

Urine and fecal samples were processed and analyzed according to the protocols of each technique $[5,23]$. To ensure quality standards, $10.0 \%$ of the slides and rapid tests were independently read by a second technician, as defined by the laboratory's current standard operational procedures.

The samples were codified and the results of the immunochromatographic test were recorded with sequential numbers per participant in order to preserve anonymity.

\section{Diagnostic methods \\ Kato-Katz}

The Kato-Katz slides [5] were prepared using the Helm Test Kit $^{\oplus}$ (Biomanguinhos, Ministry of Health, Brazil). A total of 16 slides, 12 of the first sample, two of the second one and two of the third fecal sample were used. The 12 slides from the first sample summed up to 500 mg of examined fecal matter. Together with the slides from samples two and three, a total of about $667 \mathrm{mg}$ of feces were analyzed, which, in this case, was established as the reference standard.

In addition to the reference standard, different random combinations of slides and samples were used to perform the Kato-Katz technique, as described in Fig. 2.

\section{POC-CCA}

The urine-CCA cassette is recommended for the qualitative presumptive detection of an active Schistosoma infection, being $S$. mansoni more specific. According to the manual of the producer Rapid Medical Diagnostics, the test may show false negative results in case of low infection levels. In high transmission settings, the sensitivity can reach $100 \%$ and in low transmission settings $70.0 \%$ [23].

Only one drop of first morning urine was required for the examination. The test result was reported between 20 and $25 \mathrm{~min}$ after adding samples and buffer. The blue line to test control should become pink to valid the test; any other color outside this pattern was considered invalid. All the results of the immunochromatographic test were interpreted as positive, considering the development of a second pink line, parallel to the control, otherwise the test was considered negative, according to the manufacturer recommendations (Rapid Medical Diagnostics, South Africa, 2015) [23]. It's important to note that a weak pink line, classified as 'trace' result, was

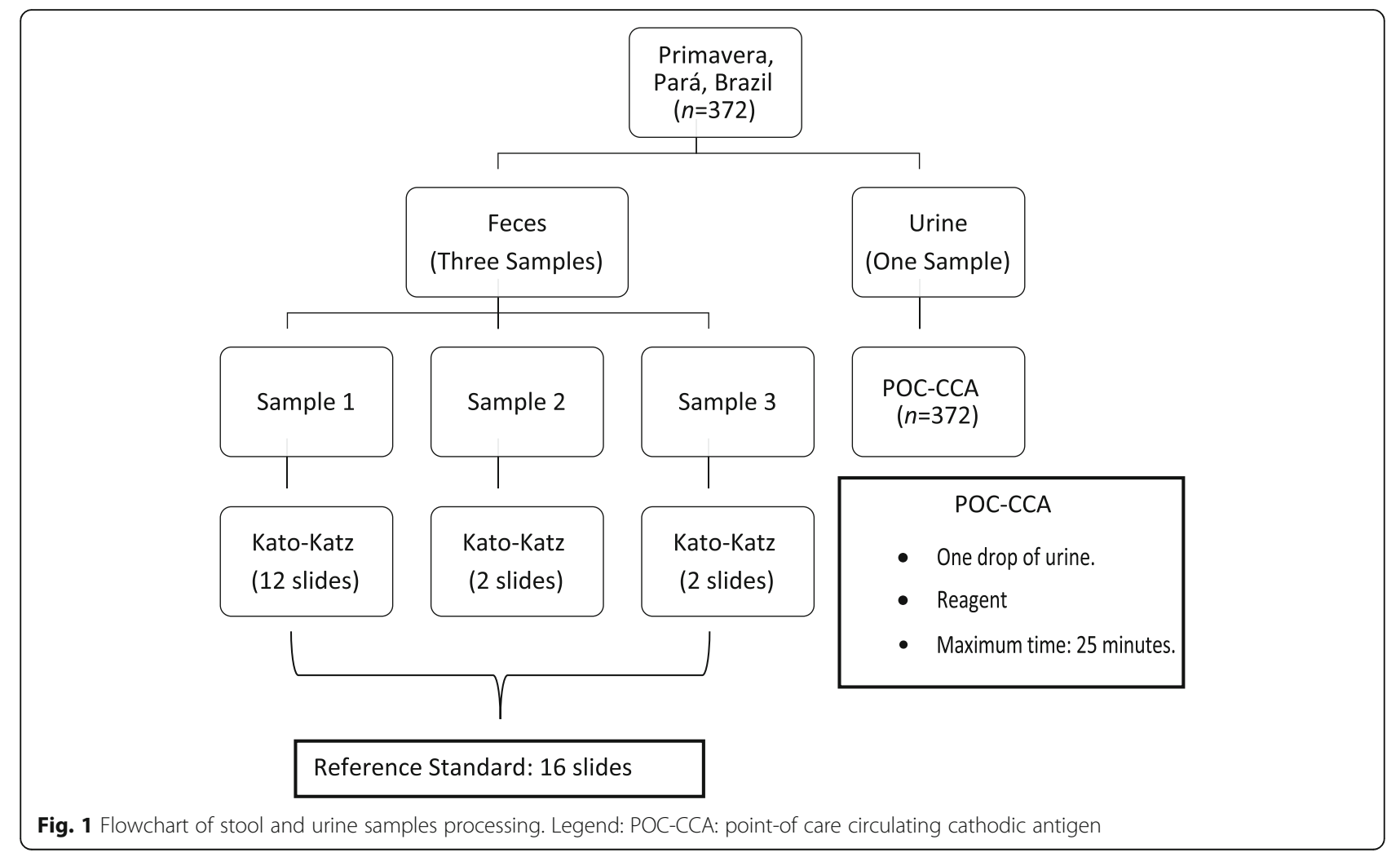




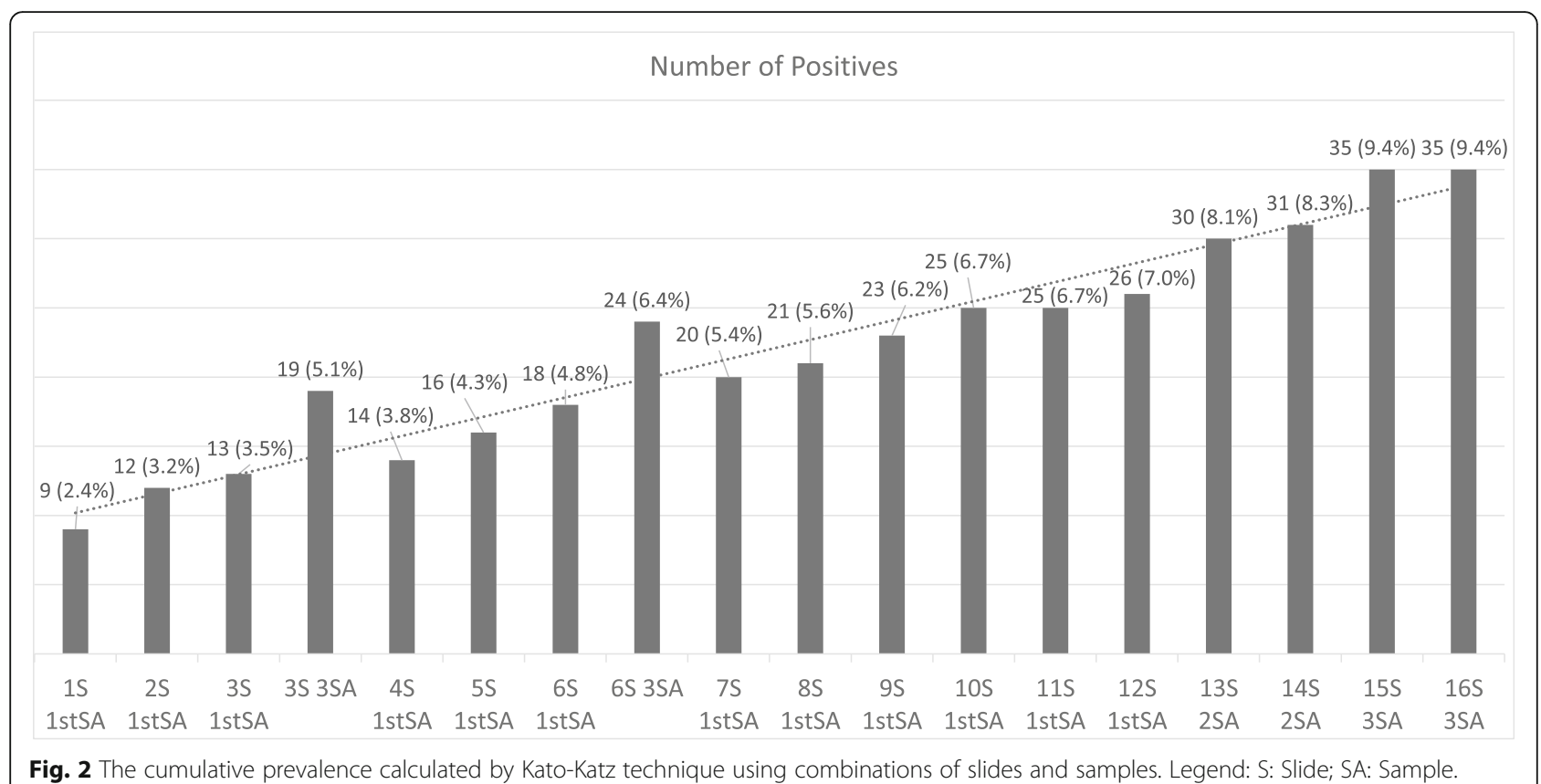

Fig. 2 The cumulative prevalence calculated by Kato-Katz technique using combinations of slides and samples. Legend: S: Slide; SA: Sample.

included into the analysis, first as positive and second as negative result, as shown in Fig. 3.

\section{Statistical analysis}

Statistical tests were performed by the online program OpenEpi version 3.01 (Open Source Epidemiologic Statistics for Public Health, available on https://www.openepi.com/Menu/OE_Menu.htm updated in 2013). The topic calculations: paired $2 \times 2$ tables in a $95 \%$ confidence interval $(C I)$. The rate of positivity, sensitivity, specificity, positive and negative predictive values and general accuracy were calculated. The kappa index was calculated to evaluate the concordance between the results obtained by the POC-CCA and the Kato-Katz method, following the classification criteria recommended by Landis \& Koch (1977) [24]. To observe statistically significant differences, the McNemar test was applied, with results considered significant if $P \leq 0.05$ and at a confidence interval of $95 \%$.

\section{Ethical considerations}

The study is part of a multicenter project, with participants from Pará, Minas Gerais and Santa Catarina. The project was submitted to the ethics committee and

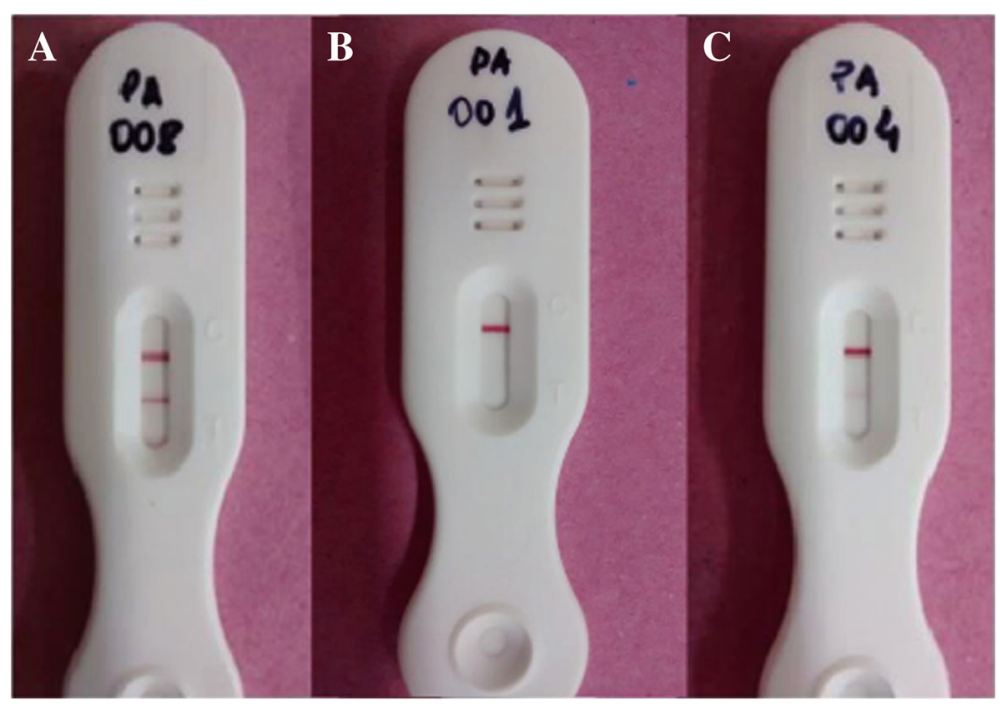

Fig. 3 Results of three POC-CCA tests. Legend: a Positive, b negative and c "Trace" 
approved with CAAE: 21824513.9.0000.5091. All participants were informed about the objectives and invited to participate voluntarily after signing the Informed Consent Form. All positive individuals according to the reference standard were treated with praziquantel, following the guidelines of the Brazilian Ministry of Health, with $60 \mathrm{mg} /$ $\mathrm{kg}$ for children and $50 \mathrm{mg} / \mathrm{kg}$ for adults.

\section{Results}

\section{Positivity rate by Kato-Katz technique}

This study consisted of 372 participants, 186 (50.0\%) male and $186(50.0 \%)$ female. The positivity rate for schistosomiasis determined by Kato-Katz technique with 16 slides was 35 (9.4\%) positive individuals. The positivity rate obtained by the Kato-Katz technique increased from 9 positives $(2.4 \%)$ to 35 positives $(9.4 \%)$, depending on the number of slides of the samples examined by participants. Sample diversification detected more positives when compared to results from one sample (Fig. 2).

Regarding to eggs count per gram (EPG) revealed by reference standard, all the individuals shown a low parasitic load. Out of 35 positive individuals, 18 had been shown two EPG. Only a single participant had a parasitic load with 332 EPG. The others 16 infected revealed an individual's count between three and 47 EPG.

About gender distribution of infection, the number of positives shown a total of 30 males infected, indicating an infection rate of $16.1 \%$ and five positive females with an infection rate of $2.7 \%$.

\section{Positivity rate by POC-CCA test}

The presence of infection in children less than 10 years old was only identified by the POC-CCA test. In relation to other age groups, the Kato-Katz technique detected more positive individuals between 21 and 40 years old as described in Table 1.

The positivity rate determined by the immunochromatographic POC-CCA test was $23.9 \%$ (89 positive individuals). It is important to emphasize that results with faint or poor visualization compared to the control range were considered "Trace", being classified as positive (Fig. 2).

\section{Accuracy analysis of the POC-CCA test}

Accuracy and matching values of the POC-CCA test results compared to the Kato-Katz technique demonstrated higher sensitivity as the number of slides increased. The sensitivity of $55.6 \%$ was observed when compared POC-CCA results with positives detected by one slide of Kato-Katz, however, it is increased to $65.7 \%$ when was compared with 16 slides of different samples (Table 2). Statistically significant differences were observed in all results of Kato-Katz slides and samples when compared with the results of the immunochromatographic test, with $P<0.0001$.

\section{"Trace" classified as negative results}

In the case that the total number of 74 POC-CCA results classified as "Traces" were considered as negative, the number of remaining positives would be reduced to 15 individuals. Table 3 provides detailed information about the positive and negative POC-CCA test results paired with the results of 16 Kato-Katz slides. It is important to note that a total of 17 results out of the 74 "Traces" considered as negative were confirmed as positive by Kato-Katz technique.

The immunochromatographic test with "Trace" classified as negative revealed sensitivity of $17.1 \%$ and specificity of $97.3 \%$. Positive and negative predictive values were 40.0 and $91.9 \%$, respectively. The kappa value was 0.19 , classified as weak concordance.

\section{Discussion}

The examination of a Kato-Katz slide is the strategy applied for the diagnosis, treatment of infected individuals and to calculate the prevalence in endemic areas by the Schistosomiasis Control Program [25]. This strategy induced a decrease in the prevalence and intensity of infection over the years, resulting in the difficulty of

Table 1 Positivity rate in different age groups revealed by the Kato-Katz technique and the rapid urine test (POC-CCA)

\begin{tabular}{|c|c|c|c|c|c|}
\hline \multirow[t]{2}{*}{ Age group } & \multirow{2}{*}{$\begin{array}{l}\text { Total } \\
\text { Number }\end{array}$} & \multicolumn{2}{|l|}{ Kato-Katz } & \multicolumn{2}{|l|}{ POC-CCA } \\
\hline & & Number of positives & $\overline{\text { Positivity rate }}$ & Number of positives & Positivity rate \\
\hline$\leq 10$ & 70 & 0 & 0 & 10 & $14.3 \%$ \\
\hline $11-20$ & 82 & 6 & $7.3 \%$ & 6 & $7.3 \%$ \\
\hline $21-30$ & 54 & 7 & $13.0 \%$ & 5 & $9.2 \%$ \\
\hline $31-40$ & 53 & 8 & $15.1 \%$ & 5 & $9.4 \%$ \\
\hline $41-50$ & 48 & 6 & $12.5 \%$ & 22 & $45.8 \%$ \\
\hline $51-60$ & 25 & 2 & $8.0 \%$ & 16 & $64.0 \%$ \\
\hline $61-70$ & 22 & 4 & $18.2 \%$ & 16 & $72.7 \%$ \\
\hline$<70$ & 18 & 2 & $11.1 \%$ & 9 & $50.0 \%$ \\
\hline
\end{tabular}

POC-CCA point-of-care circulating cathodic antigen 
Table 2 Concordance (coefficient kappa) and accuracy analysis between Kato-Katz test results obtained by an increasing number of slides and the results of one rapid urine test sample (POC-CCA)

\begin{tabular}{|c|c|c|c|c|c|c|c|c|c|}
\hline \multirow[t]{2}{*}{ POC-CCA } & \multicolumn{3}{|c|}{ Kato-Katz 1S 1st AS } & \multicolumn{3}{|c|}{ Kato-Katz 6S 3SA } & \multicolumn{3}{|c|}{ Kato-Katz 16S 3SA } \\
\hline & Positive & Negative & Total & Positive & Negative & Total & Positive & Negative & Total \\
\hline Positive & 5 & 84 & 89 & 14 & 75 & 89 & 23 & 66 & 89 \\
\hline Negative & 4 & 279 & 283 & 10 & 273 & 283 & 12 & 271 & 283 \\
\hline Total & 9 & 363 & 372 & 24 & 348 & 372 & 35 & 337 & 372 \\
\hline Sensitivity & \multicolumn{3}{|c|}{$55.6 \%$ (95\% Cl: 26.6-81.1) } & \multicolumn{3}{|c|}{ 58.3\% (95\% Cl: 38.8-75.5) } & \multicolumn{3}{|c|}{$65.7 \%$ (95\% Cl: 49.1-79.1) } \\
\hline Specificity & \multicolumn{3}{|c|}{ 76.9\% (95\% Cl: 72.2-80.9) } & \multicolumn{3}{|c|}{ 78.4\% (95\% Cl: 73.8-82.4) } & \multicolumn{3}{|c|}{$80.4 \%$ (95\% Cl: 75.8-84.3) } \\
\hline PPV & \multicolumn{3}{|c|}{$6.0 \%$ (95\% Cl: $2.4-12.5)$} & \multicolumn{3}{|c|}{ 16.0\% (95\% Cl: 9.6-24.6) } & \multicolumn{3}{|c|}{$26.0 \%$ (95\% Cl: 17.9-37.0) } \\
\hline NPV & \multicolumn{3}{|c|}{ 98.6\% (95\% Cl: 96.4-99.4) } & \multicolumn{3}{|c|}{ 96.5\% (95\% Cl: 93.6-98.1) } & \multicolumn{3}{|c|}{ 95.8\% (95\% Cl: 92.7-97.6) } \\
\hline Kappa index & \multicolumn{3}{|c|}{0.06 (95\% Cl: 0.01-0.1) } & \multicolumn{3}{|c|}{0.16 (95\% Cl: $0.1-0.2)$} & \multicolumn{3}{|c|}{0.27 (95\% Cl: $0.2-0.3)$} \\
\hline Accuracy & \multicolumn{3}{|c|}{$76.0 \%$ (95\% Cl: 74.6-82.8) } & \multicolumn{3}{|c|}{ 77.0\% (95\% Cl: 72.6-81.1) } & \multicolumn{3}{|c|}{$79.079 .0 \%$ (95\% Cl: $74.6-82.8)$} \\
\hline
\end{tabular}

Cl Confidence interval, PPV Positive predictive value, NPV Negative predictive value, S Slides, SA Sample, 1S 1st SA One slide of the first sample, 65 3SA Six slides of three different samples, two slides of each sample, 16S 3SA 16 slides of three different samples, 12 slides of the first sample, two slides of the second sample and two slides of the third sample

detecting infections with low parasite burden, since it is known that the technique has failed to detect eggs in these individuals. Consequently, it is necessary to seek for tests with an increased sensitivity, but at reasonable costs $[1,6,7,9,11,12,26]$.

In the scientific community it is well know that detection rate of positives, using the Kato-Katz technique, improves with the increases of the number of slides and stool samples examined [6-10, 26, 27]. Therefore, in this study, 16 slides from three different samples were used as reference test revealing 35 egg-positive individuals. The reference test detected 26 more positives than one Kato-Katz slide, indicating an increase of $74.0 \%$ in the detection of positives. One sample with 12 slides detected 26 positives, nine less than compared with the reference standard, resulting in a loss of $25.0 \%$ of positives detected. Using different samples, losses of positives were detected when compared to the reference standard (Fig. 2).

The low individual parasitic load found in our study indicates the difficulty in detecting infected individuals, even using 16 slides from three different samples. The more quantity of feces was used, the more egg-positive individuals were detected, as seen in Fig. 2. A

Table 3 Positive and negative results obtained with the POCCCA test considering "Traces" as negative results comparing to the Kato-Katz technique, examining 16 slides from three different samples

\begin{tabular}{llll}
\hline POC-CCA & \multicolumn{2}{l}{ Kato-Katz } & \\
\cline { 2 - 3 } & Positive & Negative & Total \\
\hline Positive & 6 & 9 & 15 \\
Negative & 29 & 328 & 357 \\
Total & 35 & 338 & 372 \\
\hline
\end{tabular}

POC-CCA Point-of-care circulating cathodic antigen test coproscopical test with higher sensitivity could be a good strategy to maximize the detection of positives. Oliveira et al. [28] conducted a research combining different parasitological techniques, such as Helmintex test, Saline gradient and Kato-Katz technique, to evaluate POC-CCA test. Their study revealed an underestimation of the "real" S. mansoni prevalence and a maximization of detection of egg-positive individuals with the combination of methods and increased diagnostic efforts.

When compared to the Kato-Katz technique, POCCCA rapid test has shown similar results, especially when applied in endemic areas with prevalence above $50.0 \%$. This result encourages the application of this rapid test for the combat against schistosomiasis, because it is sufficiently efficient, has relatively low costs and easy and fast application. However, in areas with lower prevalence, the results of the immunochromatographic test tend to show higher values than the coprology test, reaching prevalence 2.5 times higher, as seen in the study of Colley et al. and Kittur et al. [19, 20].

The present study revealed that in low prevalence areas, such as the municipality of Primavera, state of Pará, the rapid test has a positivity rate of $23.9 \%$. The prevalence of the Kato-Katz technique by the reference standard is $9.4 \%$, that is, the POC-CCA has a detection rate 2.5 times higher. In comparison with only one analyzed Kato-Katz slide, we even reached positivity rates through POC-CCA, which were up to 10 times higher. This data is often seen in studies which involve an evaluation of POC-CCA test even in different endemic setting $[19,20]$.

Studies in endemic regions with a high positivity rate have demonstrated a good performance of the immunochromatographic test when compared to Kato-Katz technique. Adriko et al. [17] evaluated the CCA in different locations in Uganda and found a sensitivity of 
$92.0 \%$ and a specificity of $50.0 \%$ of the POC-CCA test in an area with a high positivity rate $(55.0 \%)$ compared to 6 slides from three different samples of Kato-Katz. Using the same number of slides and samples in an area with a lower positivity rate $(13.0 \%)$, the sensitivity of the POCCCA test decreases to $75.0 \%$, however the specificity increases $55.0 \%$. Shane et al. [29] also observed a sensitivity of $94.2 \%$ and specificity of $59.4 \%$ of the rapid test when compared to 6 Kato-Katz slides from three different samples in a western region of Kenya, with a positivity rate of the coprology test of $38.8 \%$.

In this study, the sensitivity of the rapid test, when compared with six slides of three different samples, was 58.3\%. The decrease in sensitivity can be explained by the presence of "false negatives", with higher frequency in regions presenting individuals with low parasitic load [26]. However, Ferreira et al. [30] detected a sensitivity rate of $55.5 \%$ when POC-CCA was compared to 6 slides from three different samples. When using one Kato-Katz slide, the sensitivity of POC-CCA was $64.3 \%$. So, there was a decrease in sensitivity with increasing numbers of slides and samples, differently from that observed in our study (Table 2).

Concerning the distribution of positive and negative POC-CCA by age group, the data in Table 1 highlight that 10 positive individuals (younger than 10 years) are found, unlike what was observed in the results of Kato-Katz. The most positive age groups detected by the immunochromatographic test were from 41 to 70 years of age, but the higher positivity rate was $72.7 \%$ in individuals aged from 61 to 70 years old. In this context, the rapid test demonstrates that individuals over 41 years of age are most affected by the disease, contrasting with the Kato-Katz technique that indicates individuals over 61 years of age as the most affected.

In order to improve the sensitivity of POC-CCA test, Grenfell et al. [31] modified the urine processing and concentrated the circulating cathodic antigen by combining the test with urine filtration-concentration method. This research revealed a new way to understand "Trace" results. Nevertheless, more extensive studies in other areas with low individual parasite load are necessary to confirm these findings.

In the present study, the POC-CCA showed a higher sensitivity (65.7\%), higher kappa value (0.27), characterized as "fair", and better accuracy when compared to the reference standard. These results demonstrate the ability of the immunochromatographic test to detect positives also diagnosed by the reference test, especially in low prevalence areas. It's important to emphasize the same pattern had been found by Siqueira et al. [32] in relation to a progressive sensitivity and kappa value, however, in a high prevalence area.

Concerning the difficult visualization of some results from the immunochromatographic test, a classification characterized as "Trace" has been established, especially among individuals with low parasite load. However, some studies suggest considering "Trace" as positive, which led to the use of "weak positives" as synonyms by several authors [16, 33, 34]. In our study, the "Trace" was considered as positive result, as originally recommended by the manufacturer and the test kit imported from South Africa. Although other studies also use "Trace" results to observe intensity of infection [30, 31, 34].

However, recent publications propagate that "Traces" should be classified as negative [31, 35]. In our study, transforming 74 "Trace" results into negatives and comparing to the reference standard, a decrease in sensitivity of the POC-CCA test from $65.7 \%$ ("Trace" as positive) to $17.1 \%$ ("Trace" as negative) was found. In addition, 17 individuals diagnosed as positives by Kato-Katz technique were missed.

It is important to emphasize that the comparison of the reference standard with POC-CCA results considering "Trace" as positive; a total number of 23 true positives were detected by POC-CCA test. In contrast, considering "Trace" as negatives, a total of six true positives were identified by POC-CCA test. That means, 17 true positives would be lost. Besides that, the 23 true positives diagnosed by the immunochromatographic test were similar to the results of nine Kato-Katz slides from the same fecal sample or six slides of three different samples, which indicates, according to the data of this study, a higher sensitivity of the POCCCA when compared with two Kato-Katz slides as currently used by the governmental control program.

These findings corroborate another study, considering "Trace" results either as positive or as negative, in which "Trace" classified as positive, revealed a better performance of the test, detecting 108 infected individuals out of a population of 228 participants, with a POC-CCA test sensitivity of $64.9 \%$, specificity of $69.2 \%$, and kappa value of 0.34 . Therefore, when "Trace" was classified as negative, the sensitivity revealed low percentage of $26.8 \%$ and kappa value of 0.25 [30].

Regarding easy applicability of POC-CCA under field conditions when compared to Kato-Katz technique, a great advantage of POC-CCA is observed. When using 16 Kato-Katz slides from a total of 372 participants, 5952 slides were prepared and read for detection of $S$. mansoni eggs. With $8 \mathrm{~h}$ of daily work, 60 slides can be read within this period by a technician, taking three and a half months of work to detect $S$. mansoni infection from all participants. When applying the rapid test point-of-care circulating cathodic antigen in a urine sample, $25 \mathrm{~min}$ are required per patient to determine the outcome. A technician performed 300 tests a day, being 40 tests per hour. So, more than $80.0 \%$ of participants had been examined for schistosomiasis using the POCCCA test in just 1 day. 
In this work, we present results that should be carefully observed, since they may contribute to a better understanding in the combat against schistosomiasis in areas with low infection rates. The POC-CCA accuracy values demonstrate low sensitivity of the test in a low prevalence area, which is the focus of this study. In this initial analysis, these data do not show a satisfactory result in the use of the test as an auxiliary tool in the control of schistosomiasis in areas with low infection rates.

The presence of false negatives is an important issue that should be debated. There are a lot of reasons which could affect the detection of CCA in human urine. The most reasonable explanation mentioned in Rapid Medical Diagnostics [23] is the need about 50 worms or more to CCA can be detected. However, more methodologic studies involving CCA detection among patients with very low worm burden should be carried out to elucidate this issue.

Nevertheless, if the POC-CCA test was applied in the studied population, 89 positives would be detected. Of these, 66 will be unnecessarily treated, since the technique of Kato-Katz with 16 slides indicates absence of eggs in these individuals previously analyzed. Though, $12(34.3 \%)$ positives escape the treatment by the immunochromatographic test (Table 2). However, if a single slide of Kato-Katz technique, which is widely used to control intestinal schistosomiasis, had been applied, the data would reveal that 26 positives won't be detected, representing $74.3 \%$ of infected won't be treated (Fig. 2).

\section{Conclusions}

The best strategy which involves speed, low cost and better performance in detecting positives to schistosomiasis within the endemic area should be considered. This approach also involves the evaluation of risks and benefits administering praziquantel to individuals without infection detected by the POC-CCA test or in the loss of infected individuals not detected by a single slide of Kato-Katz technique. This study demonstrates that the use of POC-CCA test has similar detection of infected individuals when compare with 9 slides from single sample or 6 slides from 3 different samples. The increase of the sensitivity has shown a better performance from POC-CCA test according to the increase of amount of feces analyzed. Our results show poorer CCA test performance which can be justify by the very low individual egg count per gram. The impact of other issues such as urinary infection and co-infection with other helminthes on the test performance should be investigated in future studies. Thus, the immunochromatographic test has the potential to be an important tool to combat schistosomiasis because of its practicality and applicability but should be applied with caution in low prevalence areas and in programs that aim to eradicate this disease as soon as possible.

\section{Additional file}

Additional file 1: Multilingual abstracts in the five official working languages of the United Nations. (PDF $560 \mathrm{~kb}$ )

\section{Abbreviations}

CAA: Circulating anodic antigen; CCA: Circulating cathodic antigen;

Cl: Confidence Interval; EPG: Egg count per gram; POC: Point-of-care; S: Slide; SA: Sample; WHO: World Health Organization

\section{Acknowledgments}

The authors would like to thank the team of the Schistosomiasis Control Program located in Primavera for the support and the participating communities for their cooperation in this study.

\section{Funding}

Amazon Foundation for Studies and Research Support (Fapespa).

Ministry of Science, Technology and Innovation. MCTI/CNPq/MS-SCTIE-Decit n 40/2012.

\section{Availability of data and materials}

The datasets collected and/or analyzed during the study are available from the corresponding author upon reasonable request.

\section{Authors' contributions}

MJE and SMG participated in the design of the study. SRMdS, IHLD, ÁLSF, BRC, TNdCO, JFCN and MJE participated in acquisition, analysis and data interpretation and drafting of manuscript. All authors read and approved the final paper.

\section{Ethics approval and consent to participate}

The project was submitted to the ethics committee and approved, with CAAE: 21824513.9.0000.5091. Children younger than 2 years were excluded according to the study protocol. All participants signed a consent form before enrolment. Individuals diagnosed as having schistosomiasis and soiltransmitted helminth infections were treated in accordance with the Brazilian Ministry of Health guidelines.

\section{Consent for publication}

The authors agree that the copyright of the article submitted hereby, to be published in the Journal of Infectious Diseases of Poverty is transferred, throughout the world and for the full term and all extensions and renewals thereof to this journal.

\section{Competing interests}

The authors declare that they have no competing interests.

\section{Author details}

${ }^{1}$ Programa de Pós-Graduação Strictu Sensu em Biologia Parasitária na Amazônia da Universidade do Estado do Pará, Tv. Perebebuí, 2623 - Marco, Belém, PA 66087, Brazil. 'Laboratório de Parasitoses Intestinais, Esquistossomose e Malacologia (LPIEM), Secção de Parasitologia, Instituto Evandro Chagas/SVS/MS, Ananindeua, Pará, Brazil. ²Departamento de Parasitologia, Universidade Federal de Minas Gerais, Belo Horizonte, Minas Gerais, Brazil.

Received: 29 January 2019 Accepted: 16 May 2019

Published online: 30 May 2019

\section{References}

1. World Health Organization. Schistosomiasis: progress report 2001-2011 and strategic plan 2012-2020. 2013. http://apps.who.int/iris/handle/10665/78074 Accessed 05 Feb 2018

2. World Health Organization. Schistosomiasis: Key facts. 2018. http://www.who. int/news-room/fact-sheets/detail/schistosomiasis. Accessed 16 Oct 2018.

3. Noya O, Katz N, Pointier JP, Theron A, Noya BA. Schistosomiasis in America. Negl Trop Dis. 2015;2:16-7.

4. Programa de Vigilância e Controle da Esquistossomose. BR Brasília, DF. 2017. http://tabnet.datasus.gov.br/cgi/deftohtm.exe?sinan/pce/cnv/pcepe.def. Accessed 17 Sept 2018. 
5. Katz N, Chaves A, Pellegrino J. A simple device for quantitative stool thicksmear technique in schistosomiasis mansoni. Rev Inst Med Trop. 1972;14: 397-400.

6. de Vlas SJ, Gryseels B. Underestimation of Schistosoma mansoni prevalences. Parasitol Today. 1992;8(8):274-7.

7. Gryseels B, De Vlas SJ. Worm burdens in Schistosome infections. Parasitol Today. 1996;12(3):115-9.

8. Utzinger J, Booth M, Goran EKN, Muller I, Tanner M, Lengeler C. Relative contribution of day-to-day and intra-specimen variation in faecal egg counts of Schistosoma mansoni before and after treatment with praziquantel. Parasitol. 2001;122:537-44.

9. Berhe N, Medhina G, Erkoa B, Smithc T, Gedamuc S, Berededc D, et al. Variations in helminth faecal egg contours in Kato-Katz thick smears and their implications in assessing infection status with Schistosoma mansoni. Acta Trop. 2004:92(3):205-12.

10. Enk MJ, Lima AC, Massara CL, Coelho PM, Schall VT. A combined strategy to improve the control of Schistosoma mansoni in areas of low prevalence in Brazil. Am J Trop Med Hyg. 2008;78:140-6.

11. Siqueira LMV, Coelho PMZ, Oliveira AA, Massara CL, Carneiro NFF, Lima ACL et al. Evaluation of two coproscopic techniques for the diagnosis of schistosomiasis in a low-transmission area in the state of Minas Gerais, Brazil. Mem Inst Oswaldo Cruz. 2011;106:844-50.

12. Cavalcanti MG, Silva LF, Peralta RHS, Barreto MGM, Peralta JM. Schistosomiasis in areas of low endemicity: a new era in diagnosis. Trends Parasitol. 2013;29(2):75-82.

13. De Clercq D, Sacko M, Vercruysse J, Bussche W, Landouré A, Diarra A, et al. Assessment of cure by detection of circulating antigens in serum and urine, following schistosomiasis mass treatment in two villages of the office du Niger, Mali. Acta Trop. 1997;68(3):339-46.

14. Colley DG, Andros TS, Campbell CH Jr. Schistosomiasis is more prevalent than previously thought: what does it mean for public health goals, policies, strategies, guidelines and intervention programs? Infect Dis Poverty. 2017;6(1):63.

15. World Health Organization. Report of the WHO strategic and technical advisory group. In: , The eighth meeting of the strategic and technical advisory Group for Neglected Tropical Diseases (STAG-NTD): WHO Headquarters; 2015. http://www. who.int/neglected diseases/NTD_STAG_report_2015.pdf. Accessed 18 Sept 2018

16. Standley CJ, Lwambo NJS. Performance of circulating cathodic antingen (CAA) urine-dipsticks for rapid detection of intestinal schistosomiasis in school children from shoreline communities of Lake Victoria. Parasit Vectors. 2010;3:7.

17. Adriko M, Standley CJ, Tinkitina B, Tukahebwa EM, Fenwick A, Fleming FM, et al. Evaluation of circulating cathodic antinging (CCA) urine-cassette assay as a survey tool for Schistosoma mansoni in different transmission setting within Bugiri District, Uganda. Acta Trop. 2014;136:50-7.

18. Becker SL, Marti H, Zimmermann S, Vidacek D, Herrmann M, Utzinger J, et al. Application in Europe of a urine-based rapid diagnostic test for confirmation of Schistosoma mansoni infection in migrants from endemic areas. Euro Surveill. 2015;20(23):01-05.

19. Colley DG, Binder S, Campbell C, King CH, Tchuem Tchuenté LA, N'Goran EK, et al. A five-country evaluation of a point-of-care circulating cathodic antigen urine assay for the prevalence of Schistosoma mansoni. Am J Trop Med Hyg. 2013;88(3):426-32.

20. Kittur N, Castleman JD, Campbell CH Jr, King CH, Colley DG. Comparison of Schistosoma mansoni prevalence and intensity of infection, as determined by the circulating cathodic antigen urine assay or by the Kato-Katz fecal assay: a systematic review. Am J Trop Med Hyg. 2016:94(3):605-10.

21. Legesse M, Erko B. File-based evaluation of a reagent strip test for diagnosis of schistosomiasis mansoni by detecting circulating cathodic antigen (CCA) in urine in low endemic area in Ethiopia. Parasite. 2008;15(2):151-5.

22. Instituto Brasileiro de Geografia e Estatística. https://www.ibge.gov.br/ cidades-e-estados/pa/primavera.html?. Accessed 05 Feb 2018.

23. Rapid medical Diagnostics. For qualitative detection of: Bilharzia (Schistosomiasis). 2015. http://www.rapid-diagnostics.com/updates_04_02_ 2017/RMD_Pamphlet_25_01_17_Web.pdf. Accessed 06 Feb 2018.

24. Landis JR, Koch GG. The measurement of observer agreement for categorical. Biometrics. 1977;33:159-74.

25. Brasil. Vigilância da Esquistossomose mansoni: diretrizes técnicas. 4th ed. Brasília: Editora MS; 2014

26. Sousa SRM, Carvalho AQ, Cardoso JFN, Coelho PMZ, Geiger SM, Enk MJ. Schistosomiasis in the Amazon region: is the current diagnostic strategy still appropriate? Rev Soc Bras Med Trop. 2017;50(6):848-52.
27. Lovis L, Mak TK, Phongluxa K. Aye' Soukhathammavong P, Vonghachack $Y$, et al. efficacy of Praziquantel against Schistosoma mekongi and Opisthorchis viverrini: a randomized, single-blinded dose-comparison trial. PLoS Negl Trop Dis. 2012:6(7):e1726.

28. Oliveira WJ, Magalhães FC, Elias AMS, Castro VN, Favero V, Lindholz CG, et al. Evaluation of diagnostic methods for the detection of intestinal schistosomiasis in endemic area with low parasite loads: saline gradient, Helmintex, Kato-Katz and rapid urine test. PLoS Negl Trop Dis. 2018;12(2): e0006232.

29. Shane HL, Verani JR, Abudho B, Montgomery SP, Blackstock AJ, Mwinzi PNM, et al. Evaluation of urine CCA assays for detection of Schistosoma mansoni infection in Western Kenya. PLoS Negl Trop Dis. 2011;5(1):e951.

30. Ferreira FT, Fidelis TA, Pereira TH, Otoni A, Queiroz LC, Amância FF, et al. Sensitivity and specificity of the circulating cathodic antigen rapid urine test in the diagnosis of Schistosomiasis mansoni infection and evaluation of morbidity in a low-endemic area in Brazil. Rev Soc Bras Med Trop. 2017; 50(3):358-64.

31. Grenfell RFQ, Taboada D, Coutinho LA, Pedrosa MLC, Assis JV, Oliveira MSP, et al. Innovative methodology for point-of-care circulating cathodic antigen with rapid urine concentration for use in the field for detecting low Schistosoma mansoni infection and for control of cure with high accuracy. Trans R Soc Trop Med Hyg. 2018;112(1):1-7.

32. Siqueira LMV, Couto FFB, Taboada D, Oliveira AA, Carneiro NFF, Oliveira E, et al. Performance of POC-CCA ${ }^{\oplus}$ in diagnosis of schistosomiasis mansoni in individuals with low parasite burden. Rev Soc Bras Med Trop. 2016;49(3):341-7.

33. Stothard JR, Kabatereine NB, Tukahebwa EM, Kazibwe F, Rollinson D, Mathieson W, et al. Use of circulating cathodic antigen (CCA) dipsticks for detection of intestinal and urinary schistosomiasis. Acta Trop. 2006;97(2):219-28.

34. Mwinzi PNM, Kittur N, Ochola E, Cooper PJ, Campbell CH Jr, King CH, et al. Additional evaluation of the point-of-contact circulating cathodic antigen assay for Schistosoma mansoni infection. Front Public Health. 2015;3:48.

35. Coelho PMZ, Siqueira LMV, Grenfell RFQ, Almeida NBF, Katz N, Almeida A, et al. Improvement of POC-CCA interpretation by using lyophilization of urine from patients with Schistosoma mansoni low worm burden: towards an elimination of doubts about the concept of trace. PLoS Negl Trop Dis. 2016;10(6):e0004778

\section{Ready to submit your research? Choose BMC and benefit from:}

- fast, convenient online submission

- thorough peer review by experienced researchers in your field

- rapid publication on acceptance

- support for research data, including large and complex data types

- gold Open Access which fosters wider collaboration and increased citations

- maximum visibility for your research: over $100 \mathrm{M}$ website views per year

At $\mathrm{BMC}$, research is always in progress.

Learn more biomedcentral.com/submission 